\title{
Drivers of ESBL-producing Escherichia coli dynamics in calf fattening farms: A modelling study
}

\author{
Jonathan Bastard ${ }^{\text {a,b,c,d,e, }}$, Marisa Haenni ${ }^{\mathrm{f}}$, Emilie Gay ${ }^{\mathrm{g}}$, Philippe Glaser ${ }^{\mathrm{h}}$, Jean-Yves Madec ${ }^{\mathrm{f}}$, \\ Laura Temime $^{\mathrm{c}, \mathrm{d}}$, Lulla Opatowski ${ }^{\mathrm{a}, \mathrm{b}}$ \\ ${ }^{a}$ Université Paris-Saclay, UVSQ, Univ. Paris-Sud, Inserm, CESP, Anti-infective evasion and pharmacoepidemiology team, F-78180 Montigny-le-Bretonneux, France \\ ${ }^{\mathrm{b}}$ Institut Pasteur, Epidemiology and Modelling of Antibiotic Evasion unit, F-75015 Paris, France \\ ${ }^{\mathrm{c}}$ MESURS laboratory, Conservatoire national des arts et métiers, 292 rue Saint-Martin, 75003 Paris, France \\ d PACRI unit, Institut Pasteur, Conservatoire national des arts et métiers, Paris, France \\ e Université Paris Diderot, Sorbonne Paris Cité, Paris, France \\ ${ }^{\mathrm{f}}$ Université de Lyon - Anses, Laboratoire de Lyon, Unité Antibiorésistance et Virulence Bactériennes, Lyon, France \\ ${ }^{\mathrm{g}}$ Université de Lyon - Anses, Laboratoire de Lyon, Unité EAS, Lyon, France \\ ${ }^{\mathrm{h}}$ Ecology and Evolution of Antibiotics Resistance (EERA) unit, CNRS UMR 3525, Institut Pasteur, AP-HP, Université Paris-Sud, Paris, France
}

\section{A R T I C L E I N F O}

\section{Keywords:}

Antimicrobial resistance

ESBL

Livestock

Calves

Escherichia coli

Mathematical modelling

\begin{abstract}
A B S T R A C T
The contribution of bacteria in livestock to the global burden of antimicrobial resistance raises concerns worldwide. However, the dynamics of selection and diffusion of antimicrobial resistance in farm animals are not fully understood. Here, we used veal calf fattening farms as a model system, as they are a known reservoir of Extended Spectrum $\beta$-Lactamase-producing Escherichia coli (ESBL-EC). Longitudinal data of ESBL-EC carriage and antimicrobial use (AMU) were collected from three veal calf farms during the entire fattening process. We developed 18 agent-based mechanistic models to assess different hypotheses regarding the main drivers of ESBLEC dynamics in calves. The models were independently fitted to the longitudinal data using Markov Chain Monte Carlo and the best model was selected. Within-farm transmission between individuals and sporadic events of contamination were found to drive ESBL-EC dynamics on farms. In the absence of AMU, the median carriage duration of ESBL-EC was estimated to be 19.6 days (95\% credible interval: $[12.7 ; 33.3])$. In the best model, AMU was found to influence ESBL-EC dynamics, by affecting ESBL-EC clearance rather than acquisition. This effect of AMU was estimated to decrease gradually after the end of exposure and to disappear after 62.5 days [50.0; 76.9]. Moreover, using a simulation study, we quantified the efficacy of ESBL-EC mitigation strategies. Decreasing ESBL-EC prevalence by $50 \%$ on arrival at the fattening farm reduced prevalence at slaughter age by $33.3 \%$. Completely eliminating the use of selective antibiotics on arrival had a strong effect on average ESBL-EC prevalence (relative reduction of $77.0 \%$ ), but the effect was mild if this use was only decreased by $50 \%$ compared to baseline (relative reduction of $3.3 \%$ ).
\end{abstract}

\section{Introduction}

The detection of antibiotic-resistant bacteria in livestock animals has been a rising concern worldwide [1]. Extended Spectrum $\beta$-Lactamase (ESBL)-producing Enterobacterales, such as ESBL-producing E. coli (ESBL-EC), are a typical example as they are frequently reported in foodproducing animals [2], notably calves [3-6]. These bacteria have acquired resistance to most $\beta$-lactams and are responsible for severe infections in humans [7]. The importance of addressing antimicrobial resistance (AMR), and ESBL-EC in particular, with a One Health perspective is now widely recognized, given bacteria's capability to spread across human, animal and environmental sectors [8,9].

The drivers of AMR spread in livestock are not fully understood, although extensive antimicrobial use (AMU) is assumed to play a major role. Previous studies have investigated the relationship between variations in AMU and AMR in livestock at a scale ranging from an entire country [10-12] to specific farms [13-17], including cattle farms [18-22]. Some of these studies found an association between AMU and AMR, but not all of them. The reason may be that AMR prevalence on a farm not only depends on levels of exposure to antibiotics, but also relies

\footnotetext{
* Corresponding author at: Institut Pasteur, EMEA unit, 25 rue du Docteur Roux, 75015 Paris, France.

E-mail address: jonathan.bastard@pasteur.fr (J. Bastard).
} 
upon several other factors, such as importation of animals colonised with antibiotic-resistant bacteria, within-farm transmission of antibiotic-resistant bacteria between animals and/or from humans, and contamination of animals from the environment. Moreover, carriage and transmission of antibiotic-resistant bacteria are dynamic phenomena and may therefore not be well captured by classical statistical models.

Mechanistic dynamic models are useful to better understand the spread of AMR in populations [23]. They have been used extensively to study AMR spread in human populations and to assess the effect of control measures [24]. However, dynamic models simulating the transmission of AMR within farms and fitted using real longitudinal data are scarce [25-29].

Here, we propose what is, to our knowledge, the first dynamic model of AMR spread among veal calves, informed by longitudinal data on ESBL-EC carriage and AMU. Using this model, we quantitatively assess the efficacy of two different strategies to mitigate ESBL-EC prevalence on farms: decreasing ESBL-EC carriage upon arrival and decreasing AMU on fattening farms.

\section{Methods}

\subsection{Ethics}

Animal ethics approval is not required in France for rectal swabbing in calves since this is considered a non-invasive procedure. Consents were obtained from the owners of the farms.

\subsection{Study design and data collection}

The field study was led between October 2015 and March 2016 in three veal calf fattening farms located in the Brittany region (France), and referred to as farms A, B and C. As a general scheme in the veal calves industry in France, fattening farms usually rear batches of 250-300 dairy calves from 3 to 5 weeks to 5-6 months of age before slaughter. In accordance with European animal health and welfare directives 91/629/EC and 97/2/EC, calves were kept in individual pens until eight weeks of age, and then gathered in pens housing five calves until their slaughter, which was for normal sales purpose, and not specifically for the study purpose. During the fattening cycle, no new calf entered the farm.

The study design is described in [30]. In brief, within each participating farm, 50 calves of the same batch were randomly tested on arrival for ESBL-EC carriage. Swabs were streaked on selective ChromID ESBL agar (bioMérieux, Marcy l'Etoile, France) and, on each farm, the 50 calves were assigned to a positive or negative ESBL-EC status based on colony growth after $24 \mathrm{~h}$ at $37^{\circ} \mathrm{C}$. Antimicrobial susceptibility testing was performed using the disc diffusion method and ESBL production was confirmed by the double-disc synergy test. Among these 50 calves, in each farm, 10 ESBL-EC positive and 5 ESBL-EC negative calves were randomly selected, resulting in 15 calves followed longitudinally per farm, i.e. 45 calves included in the study in total. On each farm, calves were allocated to three different pens (five calves per pen) from eight weeks of age, and according to their initial ESBL-EC status: on farms B and $\mathrm{C}$, one pen gathered calves that were all initially ESBL-EC negative, while the other two pens gathered initially ESBL-EC positive calves. However, on farm A, all three pens gathered ESBL-EC positive calves because all calves tested on this farm were initially ESBL-EC positive (Supplementary Material SM1 and Fig. 1).

On all three farms, rectal swabs were then collected every two weeks from each calf on days 7, 21, 35, 49, 63, 77, 91, 106, 119, 133 and 147 after the calves' arrival, plus on day 161 for farms A and B. In total, 180 samples were collected on farms A and B, and 158 samples on farm C (SM1 and Fig. 1).

Over the study period, the antibiotics used were systematically recorded on a daily basis. Antibiotic treatments were independent from calves' ESBL-EC carriage.

\subsection{Dynamic model}

To unravel mechanisms underlying the temporal spread of ESBL-EC among calves, we built 18 variants of an agent-based discrete-time dynamic stochastic model of ESBL-EC acquisition and transmission within a calf farm. These variants included a various number of mechanisms, as described in Table 1.

In the models, at each time step $t$ (day), each calf was classified as either carrier or non-carrier of ESBL-EC (Fig. 1). Model parameters are summarized in Table 2 and the full description of the models, including equations, is provided in the SM2. Some parameters were farm-specific, while the others were common to all farms (Table 2). Models were run
Farm A

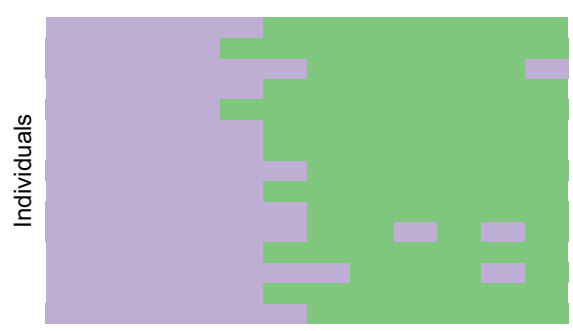

Sampling rounds

Farm C

Sampling rounds
Farm B

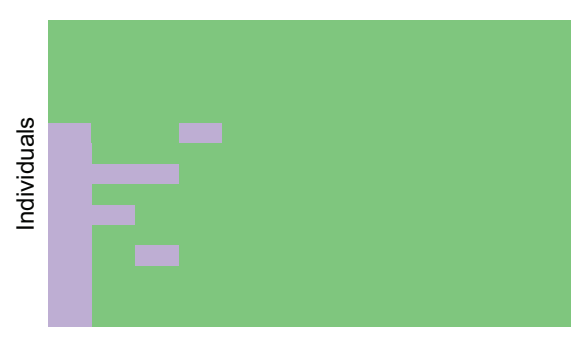

Sampling rounds
Fig. 1. Individual ESBL-EC carriage results in three calf fattening farms and model. Samples (12 in farms $\mathrm{A}$ and $\mathrm{B}$ and 11 in farm C) were collected longitudinally in 15 individuals per farm, and assigned a positive (in purple) or negative (in green) ESBL-EC status. Bottom right: dynamic model of ESBL-EC transmission in farms, where $\mathrm{A}(\mathrm{t})$ is the acquisition rate and $\mathrm{C}(\mathrm{t})$ the clearance rate for each individual calf at each time step t. (For interpretation of the references to colour in this figure legend, the reader is referred to the web version of this article.)
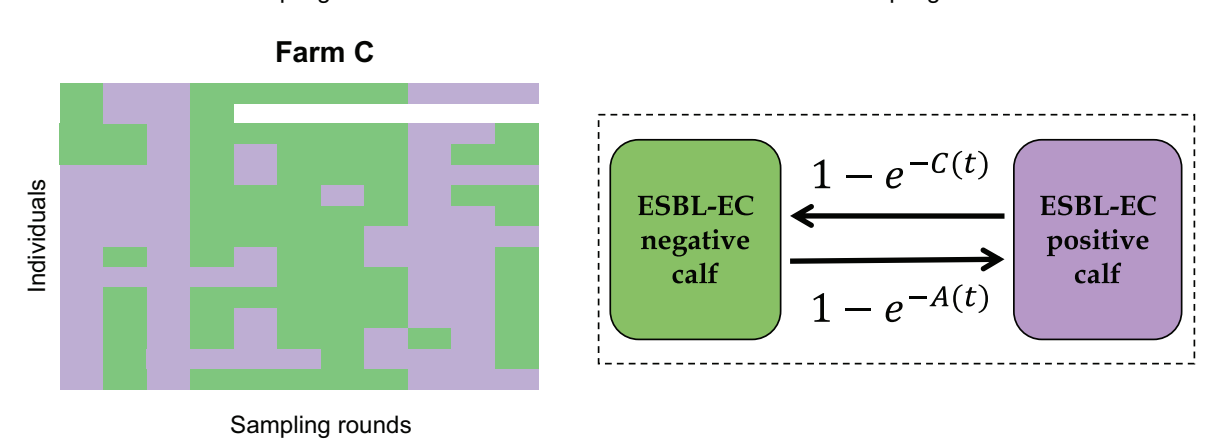
Table 1

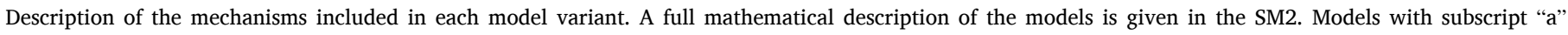
encompassed the possibility of sporadic events of contamination, while models with subscript "b" did not.

\begin{tabular}{|c|c|c|c|c|c|c|}
\hline & $\begin{array}{l}\text { Baseline constant } \\
\text { acquisition rate }\end{array}$ & $\begin{array}{l}\text { One transmission rate } \\
\text { (within farm) }\end{array}$ & $\begin{array}{l}\text { Two transmission rates on farm } \\
\text { (within pen and between pens) }\end{array}$ & $\begin{array}{l}\text { Effect of antibiotic exposure } \\
\text { on the acquisition rate }\end{array}$ & $\begin{array}{c}\text { Effect of antibiotic } \\
\text { exposure on the clearance } \\
\text { rate }\end{array}$ & $\begin{array}{l}\text { Sporadic events of } \\
\text { contamination }\end{array}$ \\
\hline $0 \mathrm{a}$ & $X$ & & & & & $X$ \\
\hline $1 \mathrm{a}$ & & $\mathrm{X}$ & & & & $\mathrm{X}$ \\
\hline $2 a$ & & & $X$ & & & $X$ \\
\hline $3 a$ & & $\mathrm{X}$ & & $\mathrm{X}$ & & $X$ \\
\hline $4 a$ & & & $\mathrm{X}$ & $\mathrm{X}$ & & $X$ \\
\hline $5 a$ & & $X$ & & & $X$ & $X$ \\
\hline $6 a$ & & & $\mathrm{X}$ & & $X$ & $X$ \\
\hline $7 a$ & $X$ & & & $X$ & & $X$ \\
\hline $8 a$ & $\mathrm{X}$ & & & & $\mathrm{X}$ & $X$ \\
\hline $0 \mathrm{~b}$ & $\mathrm{X}$ & & & & & \\
\hline $1 b$ & & $\mathrm{X}$ & & & & \\
\hline $2 b$ & & & $\mathrm{X}$ & & & \\
\hline $3 b$ & & $\mathrm{X}$ & & $\mathrm{X}$ & & \\
\hline $4 \mathrm{~b}$ & & & $\mathrm{X}$ & $X$ & & \\
\hline $5 b$ & & $X$ & & & $X$ & \\
\hline $6 \mathrm{~b}$ & & & $\mathrm{X}$ & & $X$ & \\
\hline $7 b$ & $\mathrm{X}$ & & & $\mathrm{X}$ & & \\
\hline $8 b$ & $\mathrm{X}$ & & & & $X$ & \\
\hline
\end{tabular}

\section{Table 2}

Parameters used in the dynamic models: symbol, description, unit, prior distribution and whether the parameter was farm specific (i.e. a value was estimated for each farm) or common to all farms. A specific parameter $\alpha_{\mathrm{a}, \mathrm{i}}$ (resp. $\alpha_{\mathrm{c}, \mathrm{i}}$ ) was defined for each antibiotic class i.

\begin{tabular}{|c|c|c|c|c|}
\hline Parameter & Description & Unit & $\begin{array}{c}\text { Prior } \\
\text { distribution }\end{array}$ & $\begin{array}{l}\text { Common } \\
\text { or farm- } \\
\text { specific }\end{array}$ \\
\hline$\beta_{0}^{\mathrm{F}}$ & $\begin{array}{l}\text { Constant acquisition } \\
\text { rate on farm } F\end{array}$ & $\begin{array}{l}\text { (ind. } \\
\text { day) }\end{array}$ & Uniform: $[0,10]$ & $\begin{array}{l}\text { Farm } \\
\text { specific }\end{array}$ \\
\hline$\beta_{\mathrm{f}}^{\mathrm{F}}$ & $\begin{array}{l}\text { Transmission rate } \\
\text { within the farm } F\end{array}$ & $\begin{array}{l}\text { (ind. } \\
\text { day) }\end{array}$ & Uniform: $[0,10]$ & $\begin{array}{l}\text { Farm } \\
\text { specific }\end{array}$ \\
\hline$\beta_{\mathrm{w}}^{\mathrm{F}}$ & $\begin{array}{l}\text { Transmission rate } \\
\text { within the same pen in } \\
\text { farm } F\end{array}$ & $\begin{array}{l}\text { (ind. } \\
\text { day) }\end{array}$ & Uniform: $[0,10]$ & $\begin{array}{l}\text { Farm } \\
\text { specific }\end{array}$ \\
\hline$\beta_{\mathrm{b}}^{\mathrm{F}}$ & $\begin{array}{l}\text { Transmission rate } \\
\text { between different pens } \\
\text { of the same farm } F\end{array}$ & $\begin{array}{l}\text { (ind. } \\
\text { day) })^{-1}\end{array}$ & Uniform: $[0,10]$ & $\begin{array}{l}\text { Farm } \\
\text { specific }\end{array}$ \\
\hline$\nu_{0}$ & Baseline clearance rate & day $^{-1}$ & Uniform: $[0,10]$ & Common \\
\hline$\alpha_{a, i}$ & $\begin{array}{l}\text { Effect of exposure to } \\
\text { antibiotics of class } i \text { on } \\
\text { the acquisition of } \\
\text { ESBL-EC (for a non- } \\
\text { colonised calf) }\end{array}$ & - & Uniform: $[0,10]$ & Common \\
\hline$\alpha_{c, i}$ & $\begin{array}{l}\text { Effect of exposure to } \\
\text { antibiotics of class } i \text { on } \\
\text { the clearance of ESBL- } \\
\text { EC (for a colonised } \\
\text { calf) }\end{array}$ & - & Uniform: $[0,10]$ & Common \\
\hline$\tau$ & $\begin{array}{l}\text { Rate of decrease of the } \\
\text { effect of antibiotics on } \\
\text { acquisition or } \\
\text { clearance of ESBL-EC } \\
\text { after the last day of an } \\
\text { exposure event }\end{array}$ & day $^{-1}$ & Uniform: $[0,1]$ & Common \\
\hline$N^{F}$ & $\begin{array}{l}\text { Number of sporadic } \\
\text { ESBL-EC } \\
\text { contamination events } \\
\text { during the production } \\
\text { cycle in farm } F\end{array}$ & - & $\begin{array}{l}\text { Categories of } \\
\text { equal } \\
\text { probabilities: } \\
(0,1,2)\end{array}$ & $\begin{array}{l}\text { Farm } \\
\text { specific }\end{array}$ \\
\hline$D^{F}$ & $\begin{array}{l}\text { Set of dates (days) of } \\
\text { occurrence of sporadic } \\
\text { contaminations in farm } \\
F\left(N^{F} \text { elements) }\right.\end{array}$ & - & $\begin{array}{c}\text { Categories of } \\
\text { equal } \\
\text { probabilities: }(8, \\
9, \ldots, 161)\end{array}$ & $\begin{array}{c}\text { Farm } \\
\text { specific }\end{array}$ \\
\hline$\mu$ & $\begin{array}{l}\text { Additional acquisition } \\
\text { rate due to a sporadic } \\
\text { contamination event }\end{array}$ & $\begin{array}{l}\text { (ind. } \\
\text { day) }\end{array}$ & Uniform: $[0,10]$ & Common \\
\hline
\end{tabular}

from day 1 (arrival of calves on the farm) to day 161 (last sampling date).

\subsubsection{Initialisation}

The carriage status of each calf on the first day was known from the study design described above.

\subsubsection{ESBL-EC acquisition}

At each time $t$, the probability for an ESBL-EC negative calf to acquire ESBL-EC was $1-e^{-A(t)}$, where $\mathrm{A}(\mathrm{t})$ was the acquisition rate that depended on the model variant (Fig. 1 and SM2). This acquisition could result either from transmission from other colonised calves, or from sporadic contaminations, depicting the possible acquisition of ESBL-EC by the calves on some specific days (estimated in the models) from another unknown source. Transmission was assumed to occur either homogeneously between calves of the same farm $F$, with rate $\beta_{\mathrm{f}}^{\mathrm{F}}$, or between calves depending on their allocated pen, assuming two transmission rates, within $\left(\beta_{\mathrm{w}}^{\mathrm{F}}\right)$ and between $\left(\beta_{\mathrm{b}}^{\mathrm{F}}\right)$ pens of a farm $F$. As a null hypothesis, we also investigated models which did not include any transmission between calves, but instead a constant, farm-specific, ESBL-EC acquisition rate $\beta_{0}^{\mathrm{F}}$.

\subsubsection{ESBL-EC clearance}

At each time $t$, the probability for an ESBL-EC positive calf to clear carriage was $1-e^{-C(t)}$, where $C(t)$ was the clearance rate that depended on a natural clearance rate, $\nu_{0}$, inverse of the baseline carriage duration.

\subsubsection{Impact of antibiotics}

Depending on the model variant (see Table 1), AMU was either assumed to have no effect on ESBL-EC dynamics, or to impact either the probability of acquisition or clearance (but not both simultaneously, to avoid issues of identifiability). In models $3 a, 4 a, 7 a, 3 b, 4 b$ and $7 b$, the effect was modelled on acquisition by a multiplicative factor $\lambda_{a}$ such that, at time t:

$\lambda_{a}(t)=\prod_{i} \alpha_{a, i}^{\max \left(0 ; 1-\tau E_{i}(t)\right)}$

where $\alpha_{a, i}$ represented the individual effect of $i$, a given antibiotic class, on ESBL-EC acquisition and $E_{i}(t)$ was the number of days since the end of the last exposure of the calf to this antibiotic class. After the end of exposure, this effect was supposed to persist [31], but decrease exponentially [32] (tend to 1 ) with a rate $\tau$, common to all antibiotic classes 
(see SM2 and SM7).

In models $5 \mathrm{a}, 6 \mathrm{a}, 8 \mathrm{a}, 5 \mathrm{~b}, 6 \mathrm{~b}$ and $8 \mathrm{~b}$, the effect of AMU was modelled on clearance by a similar factor:

$\lambda_{c}(t)=\prod_{i} \alpha_{c, i}^{\max \left(0 ; 1-\tau E_{i}(t)\right)}$

where $\alpha_{c, i}$ represented the individual effect of a given antibiotic class $i$ on ESBL-EC clearance, and $E_{i}(t)$ and $\tau$ were as defined above.

\subsection{Estimation and model selection}

Independently for each of the 18 models, parameters were estimated in a Bayesian framework, using a Markov Chain Monte Carlo (MCMC) algorithm, implemented with the $\mathrm{R}$ package rjags [33]. Models were fitted to the data from the three farms simultaneously. Non-informative uniform priors were used for all parameters (Table 2). For the different antibiotics, we directly used antibiotic exposure durations reported over the longitudinal study to fit the different models. The 18 models were compared using the Deviance Information Criterion (DIC) [34]. Details on modelling assumptions and estimation are provided in the SM3.

To assess the quality of the best model's fit, we simulated it by sampling parameter values in the estimated posterior distributions, and compared model predictions to observed data in each farm. In the following, the parameter values used in the model simulations are the posterior estimates from the best model.

\subsection{Simulating changes in farming practices}

We ran a simulation study to assess the impact of changes in farming practices on the mean prevalence of ESBL-EC carriage over the fattening cycle and on the final ESBL-EC prevalence at slaughter age, in farm A. We used a model without sporadic events of contamination, parameterized as described in the previous section. As a sensitivity analysis, simulations were performed in farms B and C as well.

First, we assessed the effect of exposure to "selective" antibiotics during fattening, particularly at the beginning (from day 1), as collective "starting treatments" were a common practice to manage diseases in arriving calves. Here, we defined selective antibiotics as antibiotic classes $i$ for which the estimated value of $\alpha_{a, i}$ (or $\alpha_{c, i}$ ) was significantly different from 1 in the selected model. The baseline duration of initial exposure to selective antibiotics was defined as six days, based on data from a previous representative study led in 120 French calf fattening farms [35]. Then, we simulated reductions in the duration of this initial exposure (respectively 3 days and 0 day, i.e. no exposure) to assess their effect on ESBL-EC prevalence.

Second, we evaluated the effect of ESBL-EC prevalence on arrival at the fattening farm (on day 1). Instead of using the initial ESBL-EC status of calves observed in the longitudinal study, we extracted from the literature [21] the baseline value of $68 \%$ for this prevalence on arrival in France. In the simulations, each calf therefore had this probability to be ESBL-EC positive on day 1 . We then assumed that changes in practices on dairy farms where calves were born could decrease this prevalence on arrival, and simulated such reductions by lowering this parameter's value to $0 \%$ or $34 \%$.

In all simulations, we differentiated two scenarios. In the first scenario, the only exposure to selective antibiotics was the initial exposure described above. In the second scenario, besides the initial exposure, we simulated a 10-day "mid-cycle exposure" to selective antibiotics between days 81 and 90, to mimic the treatment of diseases during fattening. Both initial and mid-cycle antibiotic exposures were simulated for all calves in the farm, irrespective of their ESBL-EC status.

\section{Results}

\subsection{ESBL-EC carriage and antimicrobial use over time}

ESBL-EC carriage of the 45 calves followed over the fattening cycle is detailed for each sampling date in the SM1 and Fig. 1. Time changes in the proportion of ESBL-EC positive calves in each farm is depicted in Fig. 2. On all three farms, the proportion of ESBL-EC positive calves was higher on the first than on the last sampling day.

On each farm, antibiotics were always administered to all calves simultaneously over the study period, i.e. there were no individual treatments. AMU observed on farms is depicted in Fig. 2 and described in the SM4.

\subsection{Parameters estimation and model comparison}

Among 18 mechanistic models, model 5a, which included farm-level between-calf transmission, impact of antibiotic exposure on carriage clearance and sporadic contaminations (Table 1) presented the lowest DIC (SM5), and was therefore selected as the best model used for all analyses onwards.

The estimated posterior distributions of model 5a parameters are summarized in Table 3 and represented in the SM6. The median posterior baseline clearance rate was 0.051 /day, corresponding to a median carriage duration of 19.6 days, in the absence of antibiotic exposure. The median farm-level transmission rate ranged between 0.021 and 0.059 (ind.day) $)^{-1}$, depending on the farm (Table 3 ).

Colistin exposure significantly affected ESBL-EC dynamics: being exposed to colistin on a given day was estimated to multiply the baseline clearance rate on that day by 0.015 (i.e. to divide it by 66.7) in median. Conversely, we did not find that the use of other antibiotic classes modified the baseline clearance: the $95 \%$ credible interval included 1 for parameters $\alpha_{c, \text { Macrolide }}, \alpha_{c, \text { Penicillin }}, \alpha_{c, \text { Sulfo.Trim. }}$ and $\alpha_{c, \text { Tetracycline. The effect }}$ of antibiotic exposure was estimated to decrease over time after the end of an antimicrobial use with a median rate of 0.016 /day, suggesting that the antibiotics affected ESBL-EC dynamics up to 62.5 days in median after the end of exposure (Table 3 and SM6\&7).

Most (69.3\%) and almost all (99.9\%) of the posterior samples in farms A and B, respectively, did not include any sporadic contamination. Conversely, there were two in farm C, at the beginning and at the end of the fattening period (Table 3 and SM6).

Model $5 \mathrm{a}$, estimated on the three farms combined, succeeded in fitting the observed data for each farm, as most of the observed data were in the 95\% prediction interval (Fig. 2). The fit of model 5a when estimated separately for each farm is shown in the SM8.

\subsection{Impact of simulated changes in farming practices}

We simulated changes in farming practices. Fig. 3 represents the mean ESBL-EC prevalence over the fattening cycle and ESBL-EC prevalence at slaughter age predicted by model $5 \mathrm{a}$ in farm $\mathrm{A}$, when three parameters vary from their baseline values. We varied: (i) ESBL-EC prevalence in calves arriving from dairy farms, (ii) the duration of calves' exposure to selective antibiotics on arrival (from day 1), and (iii) the duration of calves' exposure to selective antibiotics in the middle of the production cycle (two scenarios: 0 or 10 days from mid-cycle).

In all sets of simulations, both the mean prevalence over the cycle and prevalence at slaughter age were higher in the scenario with midcycle exposure than without. In the baseline situation corresponding to ESBL-EC prevalence on arrival and initial exposure observed in France $[21,35]$, and assuming a mid-cycle selective antibiotic exposure, the predicted median ESBL-EC prevalence at slaughter age (resp. mean prevalence over the cycle) was $20.0 \%$ (resp. 49.0\%), which was consistent with the $20.4 \%$ observed at slaughter age in [21] (Fig. 3). Therefore, in the following, we detail results only for the scenario with mid-cycle exposure, i.e. the most conservative and realistic scenario. 


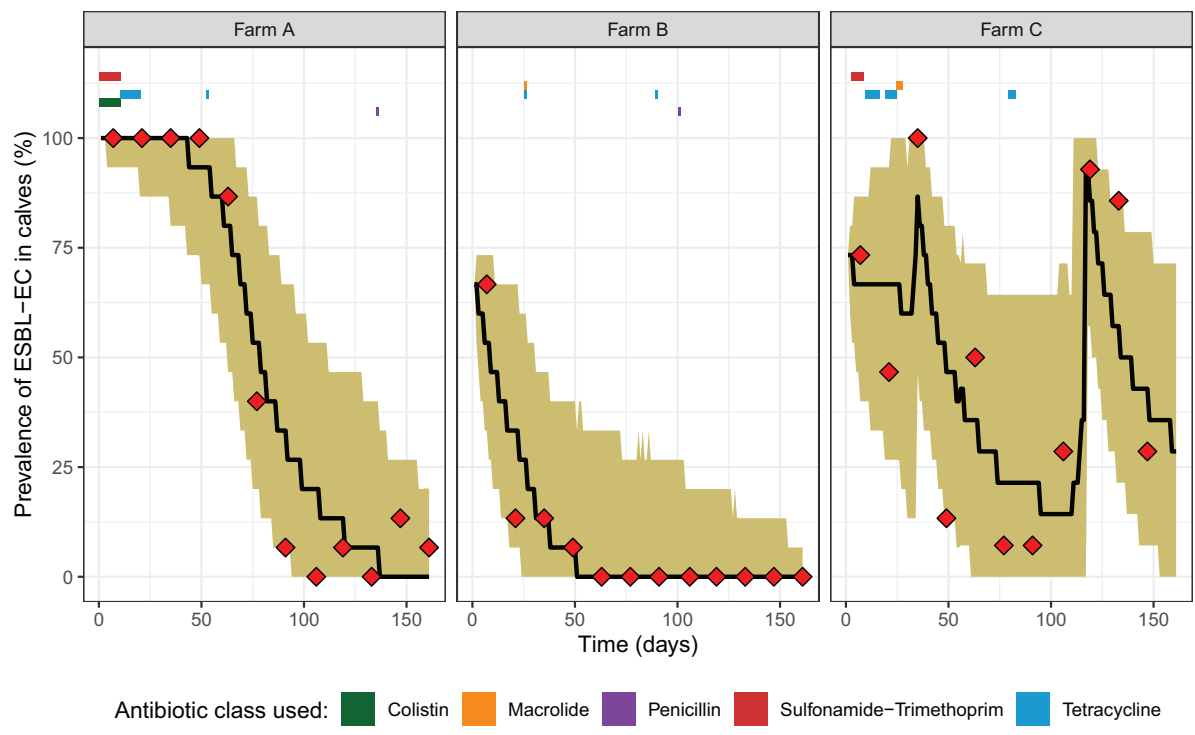

Fig. 2. Observed and predicted prevalence of ESBLEC carriage in three calf fattening farms. Samples were collected every two weeks and antibiotic usage was recorded daily (period of exposure for the different classes indicated with coloured rectangles). Observed (red diamonds), median predicted ESBL-EC prevalence (black line) and 95\% prediction interval for each farm, using model $5 \mathrm{a}$ fitted on the three farms simultaneously (5000 repetitions of the model), are represented. (For interpretation of the references to colour in this figure legend, the reader is referred to the web version of this article.)
Table 3

Posterior estimates of model 5a parameters: median and 95\% highest posterior density interval (HPDI, credible interval), or posterior distribution of the categorical variable.

\begin{tabular}{|c|c|}
\hline $\begin{array}{l}\text { Parameter } \\
\quad \text { (unit) }\end{array}$ & $\begin{array}{l}\text { Median of the posterior ( } 95 \% \text { HPDI) or Posterior distribution of the } \\
\text { categorical variable }\end{array}$ \\
\hline $\begin{array}{l}\beta_{\mathrm{f}}^{\mathrm{A}}((\text { ind. } \\
\left.\text { day })^{-1}\right)\end{array}$ & $0.021[0.0034 ; 0.052]$ \\
\hline $\begin{array}{l}\beta_{\mathrm{f}}^{\mathrm{B}}((\mathrm{ind} . \\
\left.\text { day }^{-1}\right)\end{array}$ & $0.026[0.0013 ; 0.061]$ \\
\hline $\begin{array}{r}\beta_{\mathrm{f}}^{\mathrm{C}}((\text { ind. } \\
\left.\text { day })^{-1}\right)\end{array}$ & $0.059[0.030 ; 0.093]$ \\
\hline$\nu_{0}\left(\right.$ day $\left.^{-1}\right)$ & $0.051[0.030 ; 0.079]$ \\
\hline$\alpha_{c, \text { Macrolide }}$ & $2.12[0.60 ; 4.67]$ \\
\hline$\alpha_{c, \text { Penicillin }}$ & $2.27[0.13 ; 7.85]$ \\
\hline$\alpha_{c, \text { Colistin }}$ & $0.015[0.0000026 ; 0.12]$ \\
\hline$\alpha_{c, \text { Sulfo.-Trim. }}$ & $0.46[0.095 ; 1.10]$ \\
\hline$\alpha_{c, \text { Tetracycline }}$ & $0.83[0.17 ; 2.20]$ \\
\hline$\tau\left(\right.$ day $\left.^{-1}\right)$ & $0.016[0.013 ; 0.020]$ \\
\hline$\mu$ & $6.44[2.56 ; 9.97]$ \\
\hline$N^{A}$ & 0 (69.3\%), 1 (22.1\%), 2 (8.6\%) \\
\hline$N^{B}$ & 0 (99.9\%), 1 (0.1\%), 2 (0.0\%) \\
\hline$N^{C}$ & $0(0.2 \%), 1(1.2 \%), 2(98.6 \%)$ \\
\hline
\end{tabular}

If the initial exposure to selective antibiotics was completely eliminated (resp. was reduced from 6 days to 3 days), the mean prevalence over the cycle decreased by a relative $77.0 \%$ (resp. $3.3 \%$ ) in median, from $49.0 \%$ to $11.3 \%$ (resp. $47.4 \%$ ), and the median prevalence at slaughter age was lowered to 0 (resp. was not affected) (Fig. 3B\&D).

On the other hand, if ESBL-EC prevalence on arrival was cut by half compared to the baseline, from $68 \%$ to $34 \%$, the median prevalence at slaughter age (resp. mean prevalence over the cycle) was reduced by a relative $33.3 \%$, from $20.0 \%$ to $13.3 \%$ (resp. a relative $34.8 \%$, from $49.0 \%$ to $31.9 \%$ ) (Fig. $3 \mathrm{~A} \& \mathrm{C}$ ).

The relative impacts of these different simulated changes in farming practices were qualitatively similar, no matter the farm selected for the simulations (see details in SM9).

\section{Discussion}

In this study, using longitudinal data and a dynamic model, we quantitatively estimated the between-calves transmission of ESBL-EC within farms and found a significant and persistent impact of antibiotic exposure on ESBL-EC clearance. From a simulation study, we underlined the potential impact of reductions in antimicrobial use and in ESBL-EC carriage in calves arriving from dairy farms.

Consistently with previously reported dynamics in France and the Netherlands [21,36], ESBL-EC carriage decreased from arrival to departure in all three farms (Fig. 2). Several hypotheses have been proposed to explain the high prevalence observed on arrival in fattening farms, including a strong calf-to-calf transmission during transportation or the high AMU in new-born calves [21]. The practice of using waste milk - potentially containing antibiotic residues - from treated cows in dairy farms to feed calves has also been suspected to favour an early ESBL-EC carriage [37].

Depending on the farm, the transmission rate ranged between 0.021 and 0.059 (indiv.day) $)^{-1}$, possibly reflecting differences in farm infrastructure or practices. This is in line with the ESBL-EC transmission rate of 0.06 /day estimated in broilers in the Netherlands [28]. Our median estimated carriage duration of 19.6 days was also consistent with previously reported values of 12 days for multidrug-resistant Salmonella Typhimurium in dairy cattle [38], and 26.84 days for ESBL-EC in broilers [28].

Sporadic contaminations were necessary to reproduce carriage dynamics from farm C. Such unexplained carriage increases were observed before [36]. They may reflect a contamination from the environment, companion animals, humans, or the equipment [22].

AMU patterns, with a third of treatments within the first two weeks, half of treatments by tetracycline, and a predominance of collective treatments, were similar to previous studies in French fattening calves $[21,35]$.

In the best model, AMU was shown to affect ESBL-EC clearance, rather than ESBL-EC acquisition. Among five classes of antibiotics used in the farms over the study period, we only detected a significant effect of colistin on ESBL-EC dynamics, maybe due to a lack of power for other antibiotics. For instance, the wide credible interval found for penicillin (Table 3) reflects the fact that this class was hardly used in our study.

\subsection{Main limitations of our study and perspectives}

First, we did not account for the diversity in genes conferring the ESBL phenotype and in E. coli clones. However, robust results can be drawn from modelling phenotypic AMR data alone [39]. Moreover, a mechanistic transmission model fitted with genomic data would need more complexity, accounting for (i) the within-host spread of ESBL genes between $E$. coli clones via mobile genetic elements, and (ii) the spread of $E$. coli clones between hosts. 

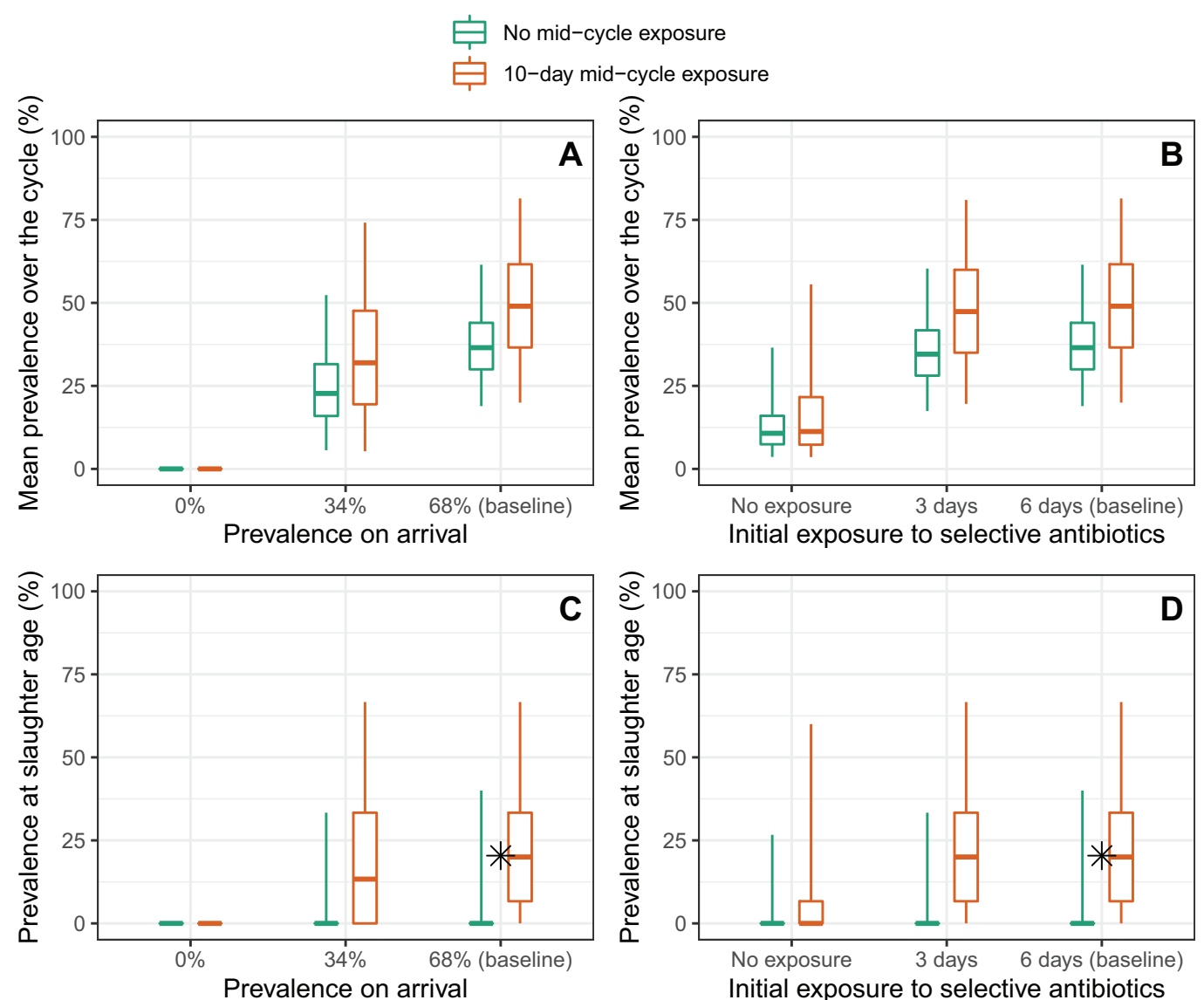

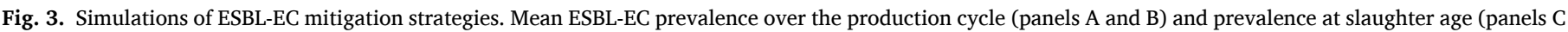

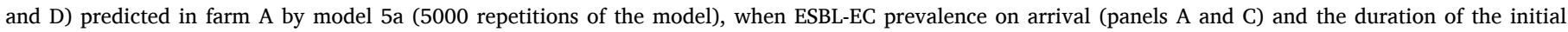

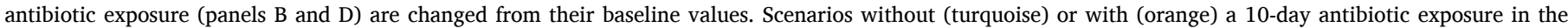

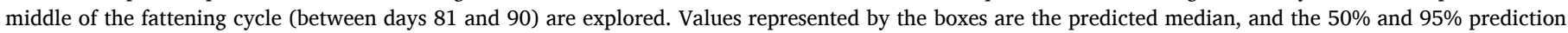

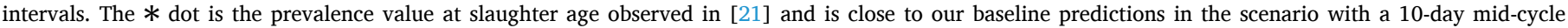
exposure. (For interpretation of the references to colour in this figure legend, the reader is referred to the web version of this article.)

Second, we assumed that antibiotic classes had an identical effect on ESBL-EC dynamics in all calves of the three farms, whereas resistance patterns in ESBL-EC strains may present individual variations. Notably, the effect of colistin on ESBL-EC we found may be specific to farm A, with colistin-resistant ESBL-EC selected in this farm only. Further genomic investigations on the presence and possible different distributions of colistin resistance genes in ESBL-EC between farms may help clarify this positive effect of colistin use on ESBL spread.

Third, we did not account for any effect of interactions between antimicrobial classes, as the scarcity of observed exposure to such combinations in our data did not allow us to assess their specific effect. Hence, the effect of colistin we found could also be attributed to the colistin-sulfonamides combination instead of colistin alone, as colistin was only used along with sulfonamides over the study period, and even though no effect was found for sulfonamide-trimethoprim.

Fourth, the effect of antibiotics was tested on acquisition or on clearance for all classes simultaneously, whereas some antibiotics may have an effect on acquisition and some others on clearance. However, this general approximation regarding the mechanism is not expected to alter our overall conclusions concerning the effect of each individual antibiotic class on ESBL-EC carriage evolution.

\subsection{Potential implications of our results for AMR mitigation}

We showed how changes in farming practices, resulting from the implementation of AMR mitigation strategies, may impact the ESBL-EC prevalence at slaughter age, reflecting the risk of its spread in the food chain, and its mean prevalence over the fattening cycle. The latter may also be of importance to human health because animals can contaminate their environment and zoonotic transmission to farmers might occur, as observed in other livestock productions [40,41].

Regarding the use of selective antibiotics during fattening, we found a contrast between the strong effect that their complete suppression had on ESBL-EC prevalence, and the mild effect found when their use was only reduced by half (Fig. 3). This non-linear effect may be explained by the persistent effect of selective antibiotics on the gut flora [42], even when they are administered for a short duration.

Reductions in ESBL-EC prevalence in calves arriving at the fattening farm simulated the hypothetical effect of actions taken at the dairy farm (e.g. reducing AMU in new-born calves or the use of waste milk from treated cows) or transportation levels (e.g. reducing calf-to-calf transmission risk). The impact of such reductions was found to be steadier, as a $50 \%$ reduction decreased by a third both the ESBL-EC prevalence at slaughter age and the average ESBL-EC prevalence over the cycle (Fig. 3).

However, to lead to field application and policy, these results would need a more thorough cost-benefit analysis of veterinary, zootechnical and economical features, along different steps of the cattle industry. In particular, calves are administered antibiotics because they are particularly susceptible to various diseases that can affect their growth and cause mortality [43,44].

Moreover, these figures correspond to a situation without sporadic 
contamination events, that can unexplainably and strongly affect ESBLEC prevalence on farms, as discussed above. This is why biosecurity is needed to reduce the potential for sporadic contamination on farms.

To estimate the implications of these results in terms of human exposure to ESBL-EC, a thorough farm-to-fork risk assessment including cross-contamination with humans, and predictive microbiology across the food processing chain, would be needed.

\section{Conclusion}

By combining the results of a longitudinal study in veal calf fattening farms and dynamic modelling, we could highlight mechanisms affecting the temporal evolution of ESBL-EC carriage in calves, and in particular the role of antimicrobials. Using stochastic simulations, we showed that minimizing prevalence upon arrival and optimizing the use of selective antibiotics during fattening are key to mitigate ESBL-EC carriage in these farms. Our methodology could be applied to other antimicrobial resistant bacteria and other livestock species.

\section{Funding}

This work was supported by the INCEPTION project (PIA/ANR-16CONV-0005) to JB, by internal resources of Institut Pasteur, the French National Institute of Health and Medical Research (Inserm) and the University of Versailles Saint-Quentin-en-Yvelines (UVSQ), by the French Government "Investissement d'Avenir" program Laboratoire d'Excellence "Integrative Biology of Emerging Infectious Diseases" (grant ANR-10-LABX-62-IBEID) and by the European Union's Horizon 2020 Research and Innovation Programme under Grant Agreement No. 773830 (Project ARDIG, EJP One Health), the TransComp-ESC-R AAP JPI-EC-AMR and INTERBEV (Protocol $N^{\circ}$ SECU-15-31). The funders had no role in study design, data collection and analysis, decision to publish, or preparation of the manuscript.

\section{CRediT authorship contribution statement}

Jonathan Bastard: Conceptualization, Formal analysis, Methodology, Software, Writing - original draft, Writing - review \& editing. Marisa Haenni: Conceptualization, Data curation, Writing - review \& editing. Emilie Gay: Conceptualization, Data curation, Writing - review \& editing. Philippe Glaser: Conceptualization, Funding acquisition, Supervision, Writing - review \& editing. Jean-Yves Madec: Conceptualization, Funding acquisition, Data curation, Writing - review \& editing. Laura Temime: Conceptualization, Funding acquisition, Methodology, Supervision, Writing - review \& editing. Lulla Opatowski: Conceptualization, Funding acquisition, Methodology, Supervision, Writing - review \& editing.

\section{Declaration of Competing Interest}

None to declare.

\section{Acknowledgements}

We sincerely thank Véronique Métayer for her technical contribution in samples management and antimicrobial susceptibility testing, and Aleksandra Kovacevic for her useful proofreading of the manuscript.

\section{Appendix A. Supplementary data}

Supplementary data to this article can be found online at https://doi. org/10.1016/j.onehlt.2021.100238.

\section{References}

[1] S.V. Puyvelde, S. Deborggraeve, J. Jacobs, Why the antibiotic resistance crisis requires a One Health approach, Lancet Infect. Dis. 18 (2) (2017) 132-134.

[2] EFSA, ECDC, The European Union Summary Report on Antimicrobial Resistance in Zoonotic and Indicator Bacteria from Humans, Animals and Food in 2016, European Food Safety Authority, 2018.

[3] J. Dantas Palmeira, H.M.N. Ferreira, Extended-spectrum beta-lactamase (ESBL)producing Enterobacteriaceae in cattle production - A threat around the world, Heliyon. 6 (1) (2020), e03206.

[4] M. Haenni, P. Châtre, V. Métayer, M. Bour, E. Signol, J.-Y. Madec, et al., Comparative prevalence and characterization of ESBL-producing Enterobacteriaceae in dominant versus subdominant enteric flora in veal calves at slaughterhouse, France, Vet. Microbiol. 171 (3) (2014) 321-327.

[5] J. Hordijk, J.A. Wagenaar, A. van de Giessen, C. Dierikx, A. van Essen-Zandbergen, K. Veldman, et al., Increasing prevalence and diversity of ESBL/AmpC-type $\beta$-lactamase genes in Escherichia coli isolated from veal calves from 1997 to 2010, J. Antimicrob. Chemother. 68 (9) (2013) 1970-1973.

[6] H.R. Springer, T.N. Denagamage, G.D. Fenton, B.J. Haley, J.A.S. Van Kessel, E. P. Hovingh, Antimicrobial resistance in fecal Escherichia coli and Salmonella enterica from dairy calves: a systematic review, Foodborne Pathog. Dis. 16 (1) (2018) 23-34.

[7] J.D.D. Pitout, P. Nordmann, K.B. Laupland, L. Poirel, Emergence of Enterobacteriaceae producing extended-spectrum $\beta$-lactamases (ESBLs) in the community, J. Antimicrob. Chemother. 56 (1) (2005) 52-59.

[8] S. Hernando-Amado, T.M. Coque, F. Baquero, J.L. Martínez, Defining and combating antibiotic resistance from One Health and Global Health perspectives, Nat. Microbiol. 4 (9) (2019) 1432-1442.

[9] J.Y. Madec, M. Haenni, P. Nordmann, L. Poirel, Extended-spectrum $\beta$-lactamase/ AmpC- and carbapenemase-producing Enterobacteriaceae in animals: a threat for humans? Clin. Microbiol. Infect. 23 (11) (2017) 826-833.

[10] F.M. Aarestrup, H. Kruse, E. Tast, A.M. Hammerum, L.B. Jensen, Associations between the use of antimicrobial agents for growth promotion and the occurrence of resistance among Enterococcus faecium from broilers and pigs in Denmark, Finland, and Norway, Microb. Drug Resist. 6 (1) (2000) 63-70.

[11] B. Callens, M. Cargnel, S. Sarrazin, J. Dewulf, B. Hoet, K. Vermeersch, et al., Associations between a decreased veterinary antimicrobial use and resistance in commensal Escherichia coli from Belgian livestock species (2011-2015), Prev. Vet. Med. 157 (2018) 50-58.

[12] L. Dutil, R. Irwin, R. Finley, L.K. Ng, B. Avery, P. Boerlin, et al., Ceftiofur resistance in Salmonella enterica serovar Heidelberg from chicken meat and humans, Canada, Emerg. Infect. Dis. 16 (1) (2010) 48-54.

[13] V.D. Andersen, L.V. De Knegt, P. Munk, M.S. Jensen, Y. Agerso, F.M. Aarestrup, et al., The association between measurements of antimicrobial use and resistance in the faeces microbiota of finisher batches, Epidemol. Infect. (2017) 2827-2837 (1469-4409 (Electronic)).

[14] J. Kylie, S.A. McEwen, P. Boerlin, R.J. Reid-Smith, J.S. Weese, P.V. Turner, Prevalence of antimicrobial resistance in fecal Escherichia coli and Salmonella enterica in Canadian commercial meat, companion, laboratory, and shelter rabbits (Oryctolagus cuniculus) and its association with routine antimicrobial use in commercial meat rabbits, Prev Vet Med. 147 (2017) 53-57.

[15] N.T. Nguyen, H.M. Nguyen, C.V. Nguyen, T.V. Nguyen, M.T. Nguyen, H.Q. Thai, et al., Use of colistin and other critical antimicrobials on pig and chicken farms in Southern Vietnam and its association with resistance in commensal Escherichia coli Bacteria, Appl. Environ. Microbiol. 82 (13) (2016) 3727-3735.

[16] K.L. Tang, N.P. Caffrey, D.B. Nóbrega, S.C. Cork, P.E. Ronksley, H.W. Barkema, et al., Restricting the use of antibiotics in food-producing animals and its associations with antibiotic resistance in food-producing animals and human beings: a systematic review and meta-analysis, Lancet Planet. Health 1 (8) (2017), e316-e27.

[17] C. Varga, A. Rajić, M.E. McFall, R.J. Reid-Smith, S.A. McEwen, Associations among antimicrobial use and antimicrobial resistance of Salmonella spp. Isolates from 60 Alberta finishing swine farms, Foodborne Pathog. Dis. 6 (1) (2008) 23-31.

[18] A.C.B. Berge, D.A. Moore, W.M. Sischo, Field trial evaluating the influence of prophylactic and therapeutic antimicrobial administration on antimicrobial resistance of fecal Escherichia coli in dairy calves, Appl. Environ. Microbiol. 72 (6) (2006) 3872

[19] B. Catry, J. Dewulf, D. Maes, B. Pardon, B. Callens, M. Vanrobaeys, et al., Effect of antimicrobial consumption and production type on antibacterial resistance in the bovine respiratory and digestive tract, PLoS One 11 (1) (2016), e0146488.

[20] S.L. Checkley, J.R. Campbell, M. Chirino-Trejo, E.D. Janzen, C.L. Waldner, Associations between antimicrobial use and the prevalence of antimicrobial resistance in fecal Escherichia coli from feedlot cattle in western Canada, Can. Vet. $\mathrm{J} .=$ La revue veterinaire canadienne 51 (8) (2010) 853-861.

[21] E. Gay, M. Bour, G. Cazeau, N. Jarrige, C. Martineau, J.-Y. Madec, et al., Antimicrobial usages and antimicrobial resistance in commensal Escherichia coli from veal calves in france: evolution during the fattening process, Front. Microbiol. 10 (2019) 792.

[22] J. Hordijk, E.A.J. Fischer, T. van Werven, S. Sietsma, L. Van Gompel, A. J. Timmerman, et al., Dynamics of faecal shedding of ESBL- or AmpC-producing Escherichia coli on dairy farms, J. Antimicrob. Chemother. 74 (6) (2019) 1531-1538.

[23] G.M. Knight, N.G. Davies, C. Colijn, F. Coll, T. Donker, D.R. Gifford, et al., Mathematical modelling for antibiotic resistance control policy: do we know enough? BMC Infect. Dis. 19 (1) (2019) 1011. 
[24] L. Opatowski, D. Guillemot, P.-Y. Boëlle, L. Temime, Contribution of mathematical modeling to the fight against bacterial antibiotic resistance, Curr. Opin. Infect. Dis. 24 (2011) 3.

[25] M. Andraud, N. Rose, M. Laurentie, P. Sanders, A. Le Roux, R. Cariolet, et al., Estimation of transmission parameters of a fluoroquinolone-resistant Escherichia coli strain between pigs in experimental conditions, Vet. Res. 42 (1) (2011) 44.

[26] E.M. Broens, C. Espinosa-Gongora, E.A.M. Graat, N. Vendrig, P.J. Van Der Wolf, L. Guardabassi, et al., Longitudinal study on transmission of MRSA CC398 within pig herds, BMC Vet. Res. 8 (2012) 58.

[27] E.M. Broens, E.A.M. Graat, A.W. van de Giessen, M.J. Broekhuizen-Stins, M.C.M. de Jong, Quantification of transmission of livestock-associated methicillin resistant Staphylococcus aureus in pigs, Vet. Microbiol. 155 (2-4) (2012) 381-388.

[28] P.M.C. Huijbers, E.A.M. Graat, A.H.A.M. van Hoek, C. Veenman, M.C.M. de Jong, E. van Duijkeren, Transmission dynamics of extended-spectrum $\beta$-lactamase and AmpC $\beta$-lactamase-producing Escherichia coli in a broiler flock without antibiotic use, Prev. Vet. Med. 131 (2016) 12-19.

[29] G. Mourand, M. Andraud, E. Jouy, C. Chauvin, L. Le Devendec, F. Paboeuf, et al., Impact of colistin administered before or after inoculation on the transmission of a mcr-1 colistin-resistant Escherichia coli strain between pigs, Vet. Microbiol. 230 (2019) 164-170.

[30] M. Massot, M. Haenni, T.T. Nguyen, J.-Y. Madec, F. Mentré, E. Denamur, Tempora dynamics of the fecal microbiota in veal calves in a 6-month field trial, Anim. Microbiome 2 (1) (2020) 32.

[31] S. Caballero, S. Kim, R.A. Carter, I.M. Leiner, B. Sušac, L. Miller, et al., Cooperating Commensals Restore Colonization Resistance to Vancomycin-Resistant Enterococcus faecium, Cell Host Microbe 21 (5) (2017) 592-602, e4.

[32] E.N. Abatih, L. Alban, A.K. Ersbøll, D.M. Lo Fo Wong, Impact of antimicrobial usage on the transmission dynamics of antimicrobial resistant bacteria among pigs, J. Theor. Biol. 256 (4) (2009) 561-573.

[33] M. Plummer, A. Stukalov, M. Denwood, M.M. Plummer, Package 'rjags', 2018.

[34] D.J. Spiegelhalter, N.G. Best, B.P. Carlin, A. Van Der Linde, Bayesian measures of model complexity and fit, J. R. Statist. Soc. B. 64 (4) (2002) 583-639.
[35] N. Jarrige, G. Cazeau, E. Morignat, M. Chanteperdrix, E. Gay, Quantitative and qualitative analysis of antimicrobial usage in white veal calves in France, Prev. Vet. Med. 144 (2017) 158-166.

[36] J. Hordijk, D.J. Mevius, A. Kant, M.E.H. Bos, H. Graveland, A.B. Bosman, et al., Within-farm dynamics of ESBL/AmpC-producing Escherichia coli in veal calves: a longitudinal approach, J. Antimicrob. Chemother. 68 (11) (2013) 2468-2476.

[37] A. Ricci, A. Allende, D. Bolton, M. Chemaly, R. Davies, P.S.F. Escámez, et al., Risk for the development of antimicrobial resistance (AMR) due to feeding of calves with milk containing residues of antibiotics, EFSA J. 15 (1) (2017), e04665.

[38] C. Lanzas, L.D. Warnick, K.L. James, E.M. Wright, M. Wiedmann, Y.T. Gröhn, Transmission dynamics of a multidrug-resistant Salmonella typhimurium outbreak in a dairy farm, Foodborne Pathog. Dis. 7 (4) (2010) 467-474.

[39] Y.T. Grohn, C. Carson, C. Lanzas, L. Pullum, M. Stanhope, V. Volkova, A proposed analytic framework for determining the impact of an antimicrobial resistance intervention, Anim. Health Res. Rev. 18 (1) (2017) 1-25.

[40] C. Dierikx, J. van der Goot, T. Fabri, A. van Essen-Zandbergen, H. Smith, D. Mevius, Extended-spectrum- $\beta$-lactamase- and AmpC- $\beta$-lactamase-producing Escherichia coli in Dutch broilers and broiler farmers, J. Antimicrob. Chemother. 68 (1) (2012) 60-67.

[41] W. Dohmen, M.J.M. Bonten, M.E.H. Bos, S. van Marm, J. Scharringa, J. A. Wagenaar, et al., Carriage of extended-spectrum beta-lactamases in pig farmers is associated with occurrence in pigs, Clin. Microbiol. Infect. 21 (10) (2015) 917-923.

[42] T. Looft, H.K. Allen, Collateral effects of antibiotics on mammalian gut microbiomes, Gut Microbes 3 (5) (2012) 463-467.

[43] C. Bähler, A. Steiner, A. Luginbühl, A. Ewy, H. Posthaus, D. Strabel, et al., Risk factors for death and unwanted early slaughter in Swiss veal calves kept at a specific animal welfare standard, Res. Vet. Sci. 92 (1) (2012) 162-168.

[44] B. Pardon, K. De Bleecker, M. Hostens, J. Callens, J. Dewulf, P. Deprez, Longitudinal study on morbidity and mortality in white veal calves in Belgium, BMC Vet. Res. 8 (2012) 26. 